\title{
CONVERSION OF LINOLEIC ACID TO DIFFERENT FATTY ACID METABOLITES BY Lactobacillus Plantarum 13-3 AND IN SILICO CHARACTERIZATION OF THE PROMINENT REACTIONS
}

\author{
TARIQ AZIZ ${ }^{a}$, ABID SARWAR ${ }^{a}$, MUHAMMAD FAHIM $^{b}$, SAM AL-DALALI ${ }^{a}$, ZIA UD DIN $^{c}$, JALAL UD DIN $^{a}$, \\ TAICIA PACHECO FILL ${ }^{d}$ AND ZHENNAI YANG ${ }^{a^{*}}$
}

\author{
${ }^{a}$ Beijing Advanced Innovation Center for Food Nutrition and Human Health, Beijing Technology and Business University, Beijing, China. \\ ${ }^{b}$ Centre for Omic Sciences, Islamia College Peshawar, KP, Pakistan 25120. \\ ${ }^{c}$ Department of Chemistry Universidade Federeal de Sao Carlos, SP, Brazil. \\ ${ }^{d}$ Institute of Chemistry Universidade Estadual de Campinas, SP, Brazil.
}

\begin{abstract}
Lactobacillus plantarum strains have been extensively used in food processing and preservation. L. plantarum also has the potential to convert polyunsaturated fatty acids, e.g. linoleic acid (LA) into bioactive and less toxic fatty acid metabolites. The objective of this study was to assess the capability of probiotic $L$. plantarum 13-3 to convert Linoleic Acid (LA) to different fatty acid metabolites in the medium supplemented with differential concentrations of LA, and the relevant reactions were characterized by in silico calculation. L. plantarum 13-3 was grown in MRS medium containing LA from 1\% to $10 \%$, and the fatty acid metabolites formed in the medium were identified and quantitated by GC-MS and in silico studies were done to confirm the enzymatic reactions involved in its conversion. The results showed that L. plantarum 13-3 could convert LA at different concentrations to 5 different fatty acid metabolites i.e, (Z)-Ethyl heptadec-9-enoate, 9,12-Octadecadienoic acid (Z, Z), methyl ester, Octadec-9-enoic acid, cis-11,14-Eicosadienoic acid, methyl ester and (Z)-18-Octadec-9-enolide. Among these metabolites, the formation of an long chain fatty acid Octadec-9-enoic Acid, also known as 18:1, N-9 or Delta(9)-Octadecenoic acid, is classified as a member of the Long-chain fatty acids in media supplemented with $4 \%$ to $10 \%$ LA, is being reported for the first time. Putative candidate enzymes involved in biotransformation of LA into fatty acid metabolites were identified in whole genome of L. plantarum 13-3, sequenced previously. In silico studies confirmed that several enzymes including the linoleate isomerase, acetoacetate decarboxylase and oxidoreductase may be involved in biotransformation of LA into fatty acid metabolites. These enzymes could effectively bind the LA molecule mainly by forming hydrogen bonding between the acidic groups of LA and the proline residues at the active sites of the enzymes validating the putative reacting partners.
\end{abstract}

Keywords: Lactobacillus plantarum, fatty acid metabolites, Linoleate isomerase, Acetoacetate decarboxylase, Dehydrogenase, in silico study.

\section{INTRODUCTION}

Linoleic acid (c18:2 n-6) (LA) the most abundant polyunsaturated fatty acid (PUFA), also known as an essential fatty acid and it is a significant constituent of low-density lipoproteins [1]. LA cannot be produced endogenously in animals and hence its only source is via dietary intake [2]. Algae and plants contain adequate amounts of the $\Delta 12$ - and $\Delta 15$-desaturase enzymes and as a result LA and $\alpha$-linolenic acid are the most widespread fatty acid found in plant tissues and oils.

As a PUFA, LA can be oxidized through endogenous enzymes and reactive oxygen species in the circulation. Metabolites of LA are formed as a result of oxidation by the action of endogenous enzymes. LA and its byproducts are known to exhibit various biological effects and they are involved in metabolic disorders and cancer [3-7]. The irony of an essential fatty acid contributing in both beneficial and pathological processes may be explained by its conversion to biologically active metabolites [8-9]. Several metabolic processes in the host are regulated by gut microbiota, some of these processes include lipid metabolism, glucose metabolism and homeostasis [10]. The gut microbiota is also capable of performing various processes that cannot be carried out by the host and these processes can give rise to microbially produced or modulated metabolites which works as metabolic substrates and signaling molecules in the host, with key implications for host metabolism and health [11]. Many bacteria have been noticed that their growth has been inhibited by Polyunsaturated fatty acids (PUFA) such as Linoleic acid (LA; 18:2 $\triangle 9 \mathrm{Z}, 12 \mathrm{Z}$ ). PUFA in high concentration is toxic and may block native fatty acid biosynthesis by the inhibition of enoyl-ACP reductase [12-15]. Bacteria have developed a detoxification mechanism and are capable of enzymatically hydrogenate PUFA by completely reducing the double bonds on the carbon chain. i.e., bio hydrogenation, producing non-toxic saturated fatty acids as the end product [16]. This process includes various steps and has been greatly described for LA and oleic acid $(\mathrm{OA} ; 18: 1 \Delta 9 \mathrm{Z})$ that are converted to non-toxic saturated stearic acid (SA; 18:0) by rumen microbiota [16-19].

Lactic acid bacteria (LAB) are the most important and common starter cultures used in fermented dairy products, and they may originate from the microflora of raw milks (e.g., bovine, ovine, caprine) but most commonly are inoculated intentionally during product manufacture.
LAB are generally regarded as safe (GRAS) microorganism which have been traditionally used in food fermentation for a long history. Traditionally fermented dairy products are considered as a key source of functional microorganism, e.g. $\mathrm{LAB}$ and ingredients [20]. Many LAB strains are able to produce various bacteriocins, exopolysaccharides, fatty acids, etc. engaging their useful health effects. Many LAB strains have shown different promising bioactivities on human health, including antimicrobial activity, prevention and treatment of diarrhea, relief of symptoms caused by lactose intolerance, anti-mutagenic and anti-carcinogenic activities and stimulation of the immune system [21]. However, the uncertainties of the influence from these LAB strains on the quality of functional foods and their bioactivity keeping in food matrix frequently prove their application in modern food industry [22].

Lactobacillus plantarum is a facultative heterofermentative lactic acid bacteria (LAB). It may get energy from different sugars, and it inhabits an adaptive nutrient-rich niche such as gastrointestinal, vaginal, and urogenital tracts, vegetables, dairy products, and fermented foods [23-28]. This bacterium also carries significant properties for not only the manufacture of a diversity of food and wine but also vitamin production, bacteriocin, probiotic, antifungal, and potential anticaries agents [29-33].

L. plantarum is homofermentative having the competency to transform growth-inhibiting free polyunsaturated fatty acids, e.g. linoleic acid (LA) at a comparatively higher concentration, into bioactive conjugated LA (CLA) and other less toxic fatty acids metabolites e.g. hydroxy fatty acids, oxo fatty acids, conjugated fatty acids and saturated trans fatty acids, as the intermediates to eventually produce saturated monoenes (OA and trans-vaccenic acid) [34-36].

In silico analysis of the whole genome sequences of L. plantarum 13-3, exposed the presence of many enzymes including acetoacetate decarboxylase, Oxidoreductase and linoleate isomerase.

We validated our findings by employing in silico analysis to characterize the relevant reactions involving the enzymes that were identified using the whole genome sequence of L. plantarum 13-3. Our findings would have great prospect for human nutrition, nutraceuticals and food industry and will pave way into identifying the relevant reactions and doses for biotransformation of LA into desirable fatty acid metabolites. 


\section{MATERIALS AND METHODS}

\section{Microorganism and growth conditions}

L. plantarum 13-3 isolated from Tibetan Kefir preserved in the culture bank of Dairy Laboratory in Beijing Technology and Business University of China was repeatedly revived for three times at $37^{\circ} \mathrm{C}$ in MRS medium (Beijing Aoboxing Co Ltd) containing $2.0 \%$ glucose, $1.0 \%$ meat extract, $1.0 \%$ tryptone, $0.5 \%$ yeast extract, $0.1 \%$ Tween $80,0.2 \% \mathrm{~K} 2 \mathrm{HPO} 4,0.5 \%$ sodium acetate, $0.2 \%$ diammonium citrate, $0.02 \%, \mathrm{MgSO} 4 \times 7 \mathrm{H} 2 \mathrm{O}$ and $0.005 \%, \mathrm{MnSO} 4 \times \mathrm{H} 2 \mathrm{O}$ was used in this study. Distilled water was used as solvent for dissolving medium components and medium was adjusted to $\mathrm{pH} 5.5$ and sterilized at $121^{\circ} \mathrm{C}$ for 15 minutes. The fresh MRS medium added with different concentrations of LA was inoculated with $1 \%$ of the activated culture of L. plantarum 13-3 for growth and production of metabolites at $37^{\circ} \mathrm{C}$.

\section{L. plantarum 13-3 Screening for the production of fatty acid metabolites}

MRS media was supplemented with linoleic acid (LA). During growth of $L$. plantarum $13-3$ in the MRS medium containing 1\%, 2\%, 3\%, 4\%, 5\%, 6\%, 7\%, $8 \%, 9 \%$ and $10 \%(\mathrm{w} / \mathrm{v})$. of LA, culture samples were taken at $0,8,16,24,36$ and $48 \mathrm{~h}$ for the determination of viable counts by plate count method as expressed by colony forming units (CFUs) per $\mathrm{mL}$, and for determination of fatty acid metabolites as explained below.

\section{Spectrophotometric determination of fatty acid metabolites}

The culture sample were centrifuged at the speed of $13,000 \times \mathrm{g}$ for $5 \mathrm{~min}$ at 4 ${ }^{\circ} \mathrm{C}$ and $1 \mathrm{~mL}$ of the supernatant was mixed with $2 \mathrm{~mL}$ of isopropanol. After addition of $1.5 \mathrm{~mL}$ of hexane, the mixture was extensively vortexed in order to extract the lipids and then allowed to stand for $5 \mathrm{~min}$. The hexane layer was collected and the absorbance was measured at $233 \mathrm{~nm}$. The fatty acid metabolites were extracted by using hexane/isopropanol $(2: 1, \mathrm{v} / \mathrm{v})$ solution at room temperature, and the extracts were washed with distilled water and then dehydrated with anhydrous sodium sulfate [37].

\section{Extraction of fatty acids from the medium.}

For the analysis by gas chromatography (GC), the culture samples were centrifuged at $1900 \mathrm{rpm}$ for 5 minutes at $4^{\circ} \mathrm{C}$ to remove the cells. An internal standard (C17:0, heptadecanoic acid, 98\% pure; Macklin) was added to $5 \mathrm{ml}$ of the supernatant fluid to give a final concentration of $0.15 \mathrm{~g} / \mathrm{ml}$. Then $5 \mathrm{~mL}$ of isopropanol was added and vortexed for $30 \mathrm{~s}$. Subsequently $2 \mathrm{~mL}$ of isopropanol was added and vortexed for $30 \mathrm{~s}$. Lastly, $5 \mathrm{~mL}$ of $\mathrm{n}$-hexane was added to this mixture, vortexed for $3 \mathrm{~min}$, incubated for $30 \mathrm{~min}$, and centrifuged at $1900 \mathrm{rpm}$ for $5 \mathrm{~min}$. The upper hexane layer containing fatty acid methyl esters (FAME) was collected and was dried under a steam of liquid nitrogen [38].

\section{Gas chromatography and mass spectrometry (GC-MS)}

The GC-MS analysis was done by using Shimadzu GC-2010 instrument coupled with a Dual Stage TMP (Ultra) mass spectrometer. The FAME sample of $2 \mu \mathrm{L}$ was injected in a split mode, set at $10: 1$ split ratio at $250^{\circ} \mathrm{C}$. The carrier gas was helium at a constant flow rate of $1 \mathrm{~mL} / \mathrm{min}$. The separation was conducted on a highly polar (TR-Wax MS, $30 \mathrm{~m}$ length $\times 0.25 \mathrm{~mm}$ i.d. $\times 0.25 \mu \mathrm{m}$ thickness) and fused silica capillary column (Thermo Fisher Scientific). The initial oven temperature was held at $170^{\circ} \mathrm{C}$ for $1 \mathrm{~min}$, then increased at $0.8^{\circ} \mathrm{C} / \mathrm{min}$ to $200^{\circ} \mathrm{C}$. The temperature of line transfer was at $250^{\circ} \mathrm{C}$, and the ion source was controlled at $200^{\circ} \mathrm{C}$. The MS detector was operated in an electron ionization (EI) voltage of $70 \mathrm{eV}$ under a mass scan range of $33-450 \mathrm{amu}(\mathrm{m} / \mathrm{z})$.

\section{Identification of fatty acid metabolites}

Chemical identification was conducted by comparison of the mass spectra (MS) of the peaks with those found in the National Institute of Standard and Technology library (NIST, 2014).

In silico characterization of conversion of $L A$ to various fatty acid metabolites by $L$. plantarum 13-3

Enzymes responsible for catalyzing the relevant reactions for conversion of LA to different fatty acid metabolites in L. Plantarum 13-3 were identified by analysis of the whole genome sequence (GCA_004028315.1) sequenced earlier using SWISS-MODEL that is a fully automated protein structure homology modelling server [39] The protein module was visualized by protein visualizing program Discovery Studio 3.5 [40]. The LA molecule was drawn in ChemSketch v15.0.9 (Chemaxon) assigned with proper 2D orientation, and the structure of each compound was analyzed for connection error in bond order. Energy of the molecules was minimized using Avogadro [41] with MMFF94 force field. Docking studies calculations were performed by Autodock Tool [42]. Protein (LAI, DH and DC) and ligands (LA) structures were converted to pdbqt file by MGL Tools 1.5.6 $\mathrm{rc}^{3}$. The interactions of complex protein-ligand conformations were analyzed using Autodock Tools 4.2 [42]. and Discovery Studio 4.1 [40].

\section{Statistical Analysis.}

Statistical analysis of the data was done using ANOVA SAS version 9 Evaluation of the significance of differences between groups was performed with one-way ANOVA as mentioned in figure legends.

\section{RESULT AND DISCUSSION}

L. plantarum 13-3 exhibited similar growth pattern at the concentrations of LA from $1 \%(\mathrm{w} / \mathrm{v})$ to $10 \%(\mathrm{w} / \mathrm{v})$ as shown in Fig. 1A. For all the concentrations of LA, it was observed that L. plantarum 13-3 increased its growth up to $24 \mathrm{~h}$, subsequently entered stationery phase till $36 \mathrm{~h}$, and then the growth decreased till $48 \mathrm{~h}$. There was slightly increase in the growth of the strain with the increase of the LA concentration. On the contrary, many other LAB strains were reported to be inhibited to different extent by LA and their tolerance to LA varied [37,3940]. While earlier studies showed that even lower LA levels $(25 \mu \mathrm{g} / \mathrm{ml})$ could inhibit bacterial growth [37], L. plantarum 13-3 was able to grow well at the concentration of LA up to $10 \%(\mathrm{w} / \mathrm{v})$, indicating its relatively high tolerance to LA. (Fig1B). showed that production of total fatty acid metabolites gradually increased with the increase of LA concentration from $1 \%(\mathrm{w} / \mathrm{v})$ to $10 \%(\mathrm{w} / \mathrm{v})$ as indicated by the increased absorption at the wavelength of $233 \mathrm{~nm}$. This indicated that $L$. plantarum 13-3 exhibited high tolerance to LA by converting LA to less toxic fatty acid metabolites. Previous researcher also advocated that conversion of free LA to LA analogues might function as a detoxification mechanism in bacteria and a stronger LA tolerance indicated a higher productivity of LA analogues/metabolites $[37,39,45-46]$. Furthermore, more fatty acid metabolites were produced during the stationery phase of growth of L. plantarum 13-3 and the production reduced when the death phase started. Similar findings were also reported with other microbial producers of LA fatty acid metabolites/analogues [47-48]. Significantly, it should be highlighted that the spectrophotometric method does not differentiate between isomers of CLA since it is based on measurement of the conjugated double bond in the fatty acid [37].

Fig $1 \mathrm{C}$ shows different fatty acid metabolites produced by $L$. plantarum 13-3 with different concentrations of LA in the medium with different time interval of $8,16,24,36$ and 48 hours.

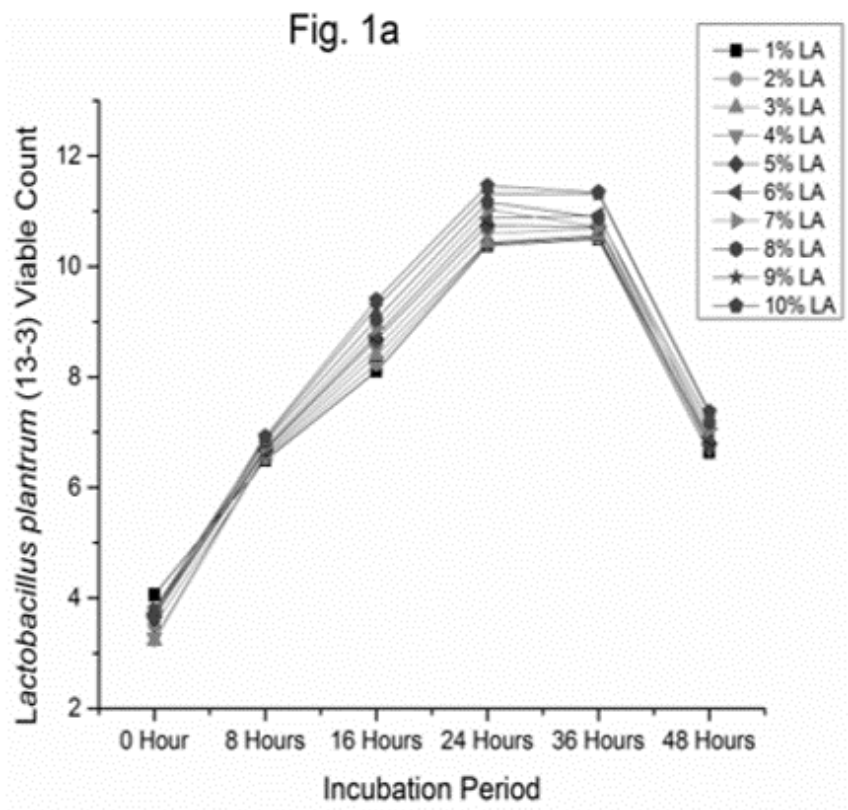


Fig. 1b
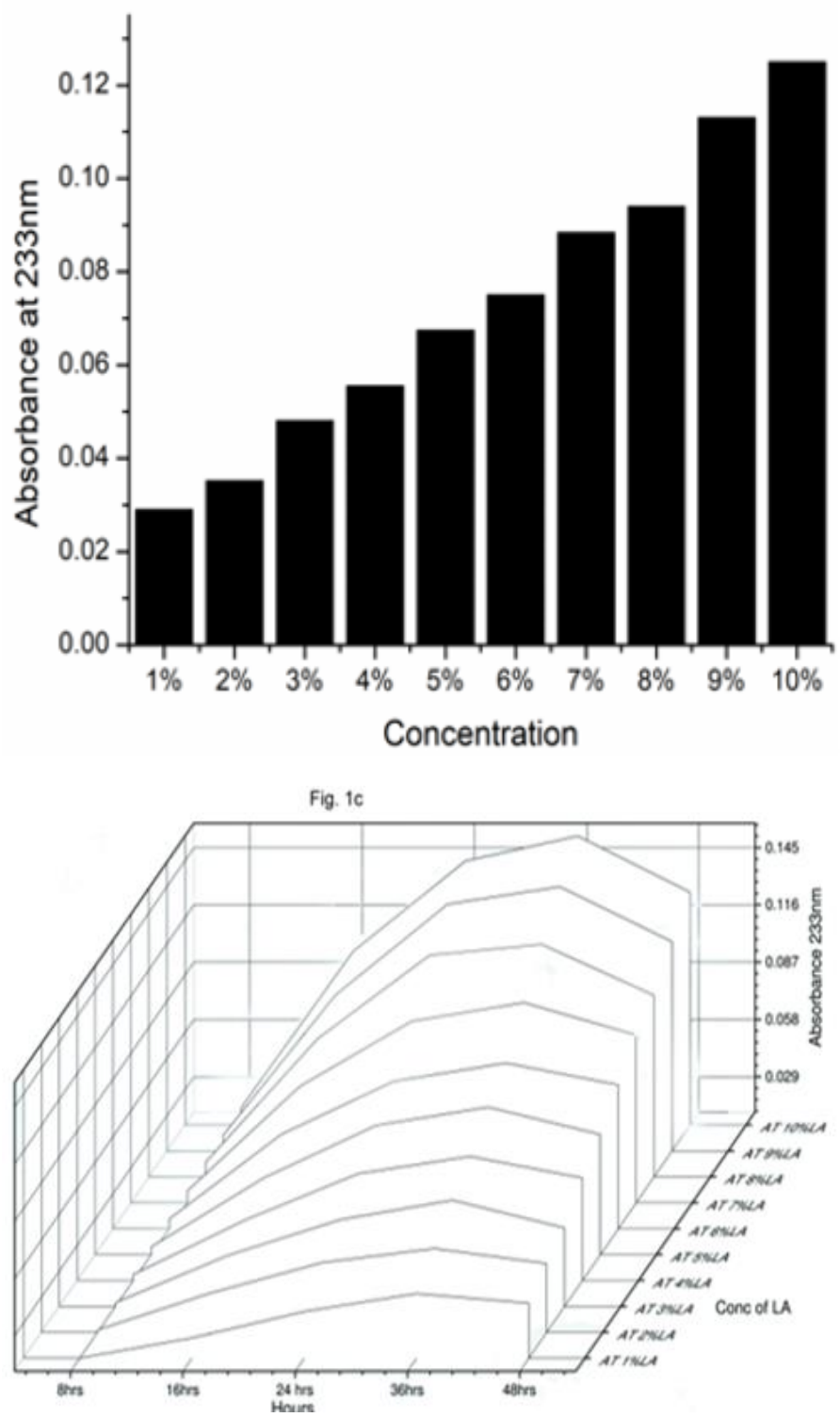

Figure 1. Lactobacillus plantarum 13-3 and production of fatty acid metabolites: (1.a.). Growth pattern of $L$. plantarum 13-3 as affected by different concentrations of linoleic acid showing plate count method at $0,8,16,24,36$ and $48 \mathrm{~h}$ respectively. (1.b.) fatty acid metabolites produced by $L$. plantarum 13-3 with different concentrations of LA in the medium as determined by spectrophotometric method at absorbance of $233 \mathrm{~nm}$. (1.c.) fatty acid metabolites produced at $8,16,24,36$ and $48 \mathrm{~h}$ during the growth of L. plantarum 13-3 with different concentrations of LA in the medium.

\section{Identification of fatty acid metabolites by GC-MS}

The fatty acid metabolites produced by $L$. plantarum $13-3$ at different concentrations of LA were identified and quantitated by GC-MS (Table 1). Total of 5 fatty acid metabolites were identified including one long chain fatty acid, octadec-9-enoic acid $\mathrm{C}_{18} \mathrm{H}_{34} \mathrm{O}_{2}$, one ethyl ester, (Z)-ethyl heptadec-9-enoate $\mathrm{C}_{19} \mathrm{H}_{36} \mathrm{O}_{2}$, two methyl esters 9,12-octadecadienoic acid (Z,Z)- methyl ester $\mathrm{C}_{19} \mathrm{H}_{34} \mathrm{O}_{2}$ also known as methyl linoleate which is found in clove and is mainly used as a flavoring agent and cis-11,14-eicosadienoic acid, methyl ester, and (Z)18-octadec-9-enolide as shown in Table 1. The following compounds, (Z)-ethyl heptadec-9-enoate, 9,12-octadecadienoic acid (Z, Z)- methyl ester, and cis11,14-eicosadienoic acid methyl ester were identified under the all concentration of added LA as shown in Table 1. These findings proved that the ability of $L$. plantarum 13-3 to produce the above mentioned fatty acids. While, the octadec-9-enoic acid was produced by $L$. plantarum 13-3 under the concentration of added LA ranged from $4 \%$ to $10 \%$. Finally, (Z)-18-octadec-9-enolide was produced only at $10 \%$ of added LA as listed in Table 1 .

\begin{tabular}{|c|c|c|c|c|c|c|c|c|c|c|c|}
\hline Name & RT & $1 \%$ & $2 \%$ & $3 \%$ & $4 \%$ & $5 \%$ & $6 \%$ & $7 \%$ & $8 \%$ & $9 \%$ & $10 \%$ \\
\hline (Z)-Ethyl heptadec-9-enoate & 15.112 & + & + & + & + & + & + & + & + & + & + \\
\hline $\begin{array}{l}\text { 9,12-Octadecadienoic acid } \\
\text { (Z,Z)-, methyl ester }\end{array}$ & 15.729 & + & + & + & + & + & + & + & + & + & + \\
\hline Octadec-9-enoic acid & 29.670 & - & - & - & + & + & + & + & + & + & + \\
\hline $\begin{array}{l}\text { cis-11,14-Eicosadienoic acid, } \\
\text { methyl ester }\end{array}$ & 31.935 & + & + & + & + & + & + & + & + & + & + \\
\hline (Z)-18-Octadec-9-enolide & 32.002 & - & - & - & - & - & - & - & - & - & + \\
\hline
\end{tabular}

+ detected, - not detected

\section{In silico studies for relevant reactions}

\section{Linoleate Isomerase}

Linoleate isomerase is the enzyme which has the responsibility for the conversion of one isomeric form of ligand to another form via enzymatic mechanism. Isomers are different form of the same ligands having same molecular formula but different structural formula having different 3D orientation in space. The genome of $L$. plantarum 13-3 was blasted to discourse different linoleate isomerase giving many hit, and best hit was selected for further study.

The Conversion of linoleic acid (LA) into several metabolites were studied by using computational approach. LA was optimized by using Avogadro and saved into PDB format. Linoleate isomerase gene was found in L. plantarum 13-3 which was blasted by using NCBI platform and then module by using Swiss model. Linoleate isomerase is a monomer protein having tertiary structure which consists of its backbone, side chain, hydrophobic moiety, hydrophilic moiety, acidic group and basic group shown in figure 2 . The collective interaction of the linoleate isomerase with LA was carried by using Auto-dock Vina Program. The optimized structure of linoleate isomerase and linoleic acid was used for Autodock study.

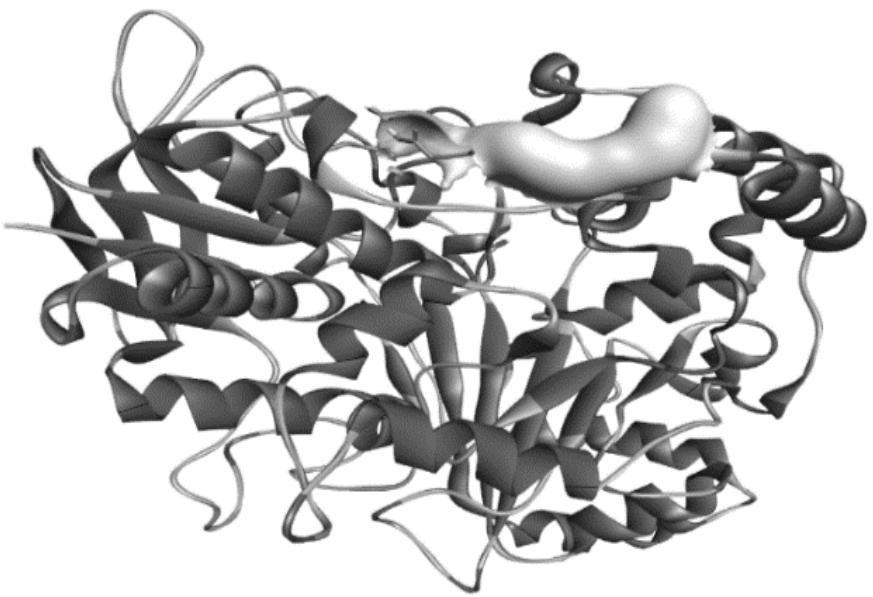

Figure 2. showing the structure of enzyme Linoleate isomerase in $L$. plantarum 13-3.

The enzyme Linoleate Isomerase of 13-3 consists of monomer having active site amino acid include lysine 549, Valine 548, Arginine 530, Leucine 547, Methionine 544, Proline 551, Threonine 550, Arginine 552, Lysine 555 and Aspartate 556. The Hydrogen bond between Lysine 549 and LA have a distance of $1.954 \mathrm{~nm}$ while the angle DHA (Donor Hydrogen Atom) is 176.65 and angle HAY (Hydrogen Acceptor) is 139.82.

The following table is showing the docking result of LA with Linoleate Isomerase (13-3). 
Table 2. Interaction of LA with Linoleate isomerase, and their result calculated by autodock.

\begin{tabular}{|l|c|}
\hline Binding Energy & -1.89 \\
\hline Ligand efficiency & -0.09 \\
\hline Inhibition Constant & 41.27 \\
\hline Intermol Energy & -5.61 \\
\hline Vdw_hb_desolv energy & -4.94 \\
\hline Electrostatic energy & -0.67 \\
\hline Total_internal & -0.4 \\
\hline Calculated RMS & 0.0 \\
\hline
\end{tabular}

After docking it was observed that Carboxylic Acidic moiety of LA formed Hydrogen bonding with Lysine (LYS A:549) and also showed electrostatic and Vander wall interactions. As Linoleate isomerase is NADPH dependent enzyme so Hydrogen from NADPH attack on the double bond of the LA ultimately the reaction of LA with Linoleate Isomerase and NADPH leading to the formation of different metabolites.

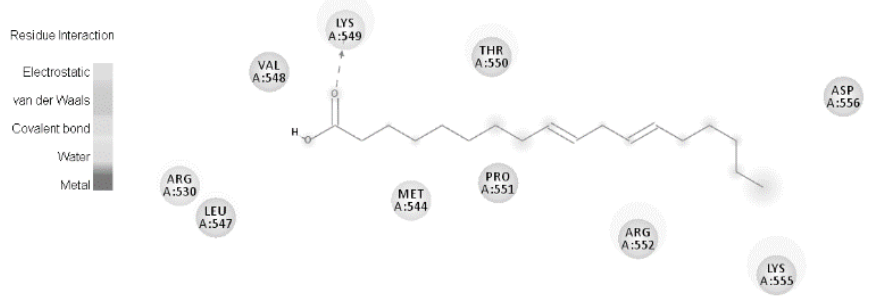

Figure 3: 2D Interaction of LA with Linoleate Isomerase, shows the 2D interaction of Linoleic Acid with the enzyme Linoleate Isomerase.

The interaction of the LA in the active site of the Linoleate Isomerase showing the Perfect fit because it occupies the active site of the Linoleate Isomerase which provides the pathway for enzymatic reaction.

The Acidic group of LA formed hydrogen bond with Lysine 549 (LYS:549). The acidic moiety holds by enzyme by hydrogen and electrostatic interaction, which provide medium for the double bond transfer reaction.

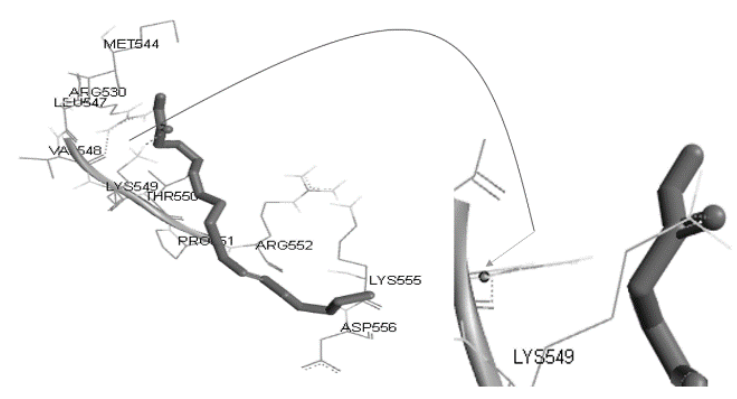

Figure 4. 3D interaction of the LA in the active site of Linoleate Isomerase.

\section{Acetoacetate Decarboxylase}

Acetoacetate decarboxylase belongs to the class of enzymes which plays a vital role in solvent production by catalyzing the decarboxylation of acetoacetate moieties, producing acetone and carbon dioxide. The production of solvent acetone by enzyme acetoacetate decarboxylase containing bacteria was utilized in large-scale industrial syntheses in the first half of the twentieth century. Recently we have explored in this study that beyond it, it also plays important role in fatty acid chemistry by converting LA into different fatty acid metabolites.

The genome of $L$. plantarum 13-3 was blasted for the search of different Acetoacetate Decarboxylase giving many hit, and best hit was selected for further study. The Protein Structure of Acetoacetate Decarboxylase of L. plantarum 133 module by Swiss model is shown in figure below. The structure of enzyme Acetoacetate Decarboxylase having ligand in the active site is given below in figure 5 .

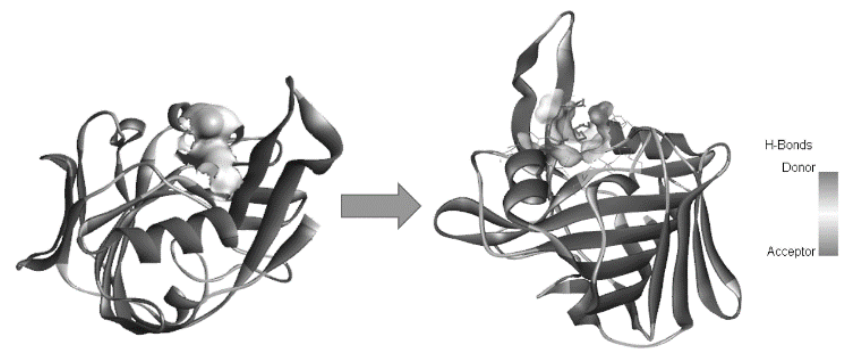

Figure 5. showing the structure of enzyme acetoacetate decarboxylase in $L$. plantarum 13-3 and Hydrogen bonding.

After docking it was observed that Carboxylic Acidic moiety of LA formed two Hydrogen bonding with Lysine (LYS A:114) and Arginine (AGR:106) and also showed electrostatic and Vander wall interactions.

The enzyme Acetoacetate Decarboxylase of L. plantarum 13-3 is an oligomer which consists of twelve monomer having active site amino acid include lysine 114, Arginine 106, Isoleucine 179, Tyrosine 177, Tyrosine 87, Leucine 115 , Aspartate 219 and Aspartate 220. The Hydrogen bond between Arginine 106 and LA have a distance of $2.233 \mathrm{~nm}$ while the angle DHA (Donor Hydrogen Acceptor) is 159.62 and angle HAY (Hydrogen Acceptor on Y Axis) is 145.70 and LA having the distance of $2.97 \mathrm{~nm}$ has the DHA angle 98.73 and the HAY angle 165.99. The second hydrogen bond between Lysine 114 and LA have a distance of $2.314 \mathrm{~nm}$ while the angle DHA (Donor Hydrogen Atom) is 151.04 and angle HAY (Hydrogen Acceptor) is 106.001.

The following table 3 is showing the docking result of LA with Acetoacetate Decarboxylase L. plantarum 13-3.

Table 3. Interaction of LA with acetoacetate decarboxylase, and their result calculated by autodock.

\begin{tabular}{|l|c|}
\hline Binding Energy & -2.31 \\
\hline Ligand efficiency & -0.12 \\
\hline Inhibition Constant & $20.28 \mathrm{mM}$ \\
\hline Intermol Energy & -5.41 \\
\hline Vdw_hb_desolv energy & -4.54 \\
\hline Electrostatic energy & -0.87 \\
\hline Total_internal & -1.02 \\
\hline Calculated RMS & 0.0 \\
\hline
\end{tabular}

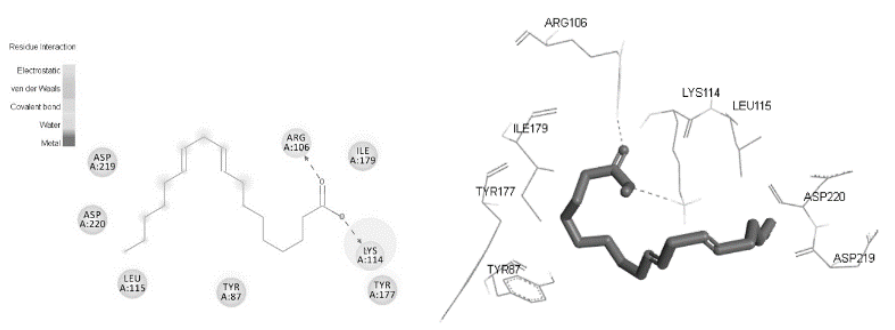

Figure 6. 2D and 3D Interaction of LA with Acetoacetate Decarboxylase

Figure shows 2D and 3D interaction of Linoleic Acid with the enzyme Acetoacetate Decarboxylase in L. plantarum 13-3.

The interaction of the LA in the active site of the Acetoacetate Decarboxylase showing the Perfect fit because it occupies the active site of the Acetoacetate Decarboxylase which provides the pathway for enzymatic reaction.

The Acidic group of LA formed two hydrogen bonds with Lysine 114 (LYS: A114) and the other with Arginine 106 (Agr: A106). The acidic moiety holds by enzyme by hydrogen and electrostatic interaction, which provide medium for the double bond transfer reaction. 


\section{Oxidoreductase}

Oxidoreductase is a class of enzyme that are involved in catalyzing the reaction which involve the transfer of electrons from one molecule, the reductant, also called the electron donor, to another, the oxidant, also called the electron acceptor. So these enzymes usually involve the transfer of charge from catalyzing molecule leading to oxidation or reduction. This group of enzymes usually NADP or NAD+ dependent enzyme which use it as cofactors. Dehydrogenase is tetramer, composed of monomer A, B, C and D. The active site residue and substrate in it is reported in figure 7 given below.

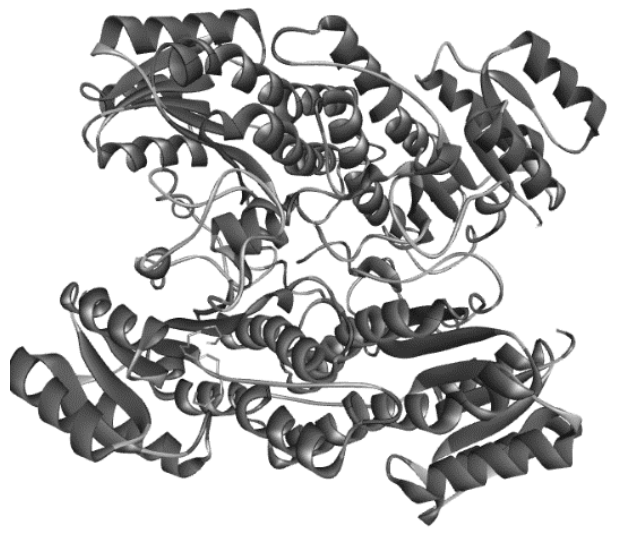

The active site residue and substrate in it is reported in the following figures. The amino acids in the active site are Glycine 17, Tyrosine 206, Asparagine 90, Methionine 186, Glycine 189, Tyrosine 156, Glycine 92, Isoleucine 93, Aspargine 114 and Lysine 160. The amino acid GLY A:17 in the active site of oxidoreductase form hydrogen bonding with substrate (LA), Which provide the plate form for the enzymatic reaction by holding acidic moiety of substrate for reduction reaction. As these enzymes are cofactor dependent enzyme, so hydrogen from the FADH or NADPH attack on the unsaturated moiety and resulting the formation of reduction product. Moreover, the docking result is reported in table 4.

The following table 4 is showing the docking result of LA with Oxidoreductase in L. plantarum 13-3.

\begin{tabular}{|l|c|}
\hline Binding Energy & -1.64 \\
\hline Ligand efficiency & -0.08 \\
\hline Inhibition Constant & 62.68 \\
\hline Intermol Energy & -4.98 \\
\hline Vdw_hb_desolv energy & -5.29 \\
\hline Electrostatic energy & -0.31 \\
\hline Total_internal & -0.78 \\
\hline Calculated RMS & 0.0 \\
\hline
\end{tabular}

The Hydrogen bond between Lysine 17 and LA have a distance of $2.38 \mathrm{~nm}$ while the angle DHA (Donor Hydrogen Atom) is 130.161 and HAY (Hydrogen Acceptor) angle is 91.442 .

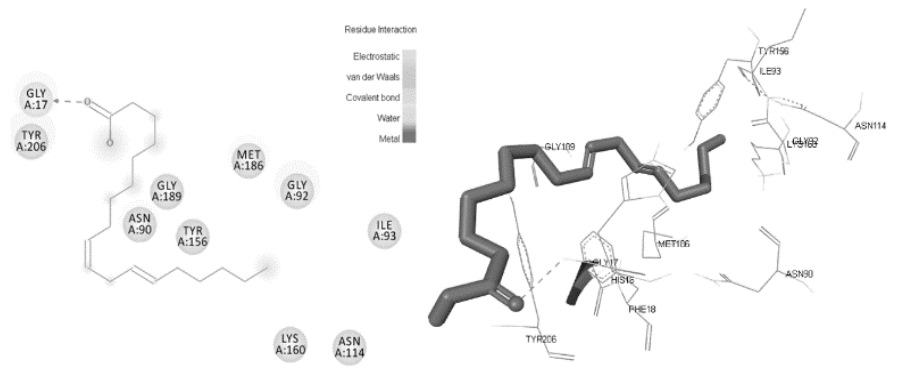

Figure 8. 2D and 3D interaction of LA with the enzyme Oxidoreductase in $L$. plantarum 13-3, is showing the 2D and 3D interaction of Linoleic acid with the enzyme Dehydrogenase Oxidoreductase in L. plantarum 13-3.

\section{CONCLUSION}

L. plantarum 13-3 was shown to be a competent candidate for converting LA from $1 \%$ to $10 \%(\mathrm{w} / \mathrm{v})$ to various fatty acid metabolites such as Z)-Ethyl heptadec-9-enoate, 9,12-Octadecadienoic acid (Z,Z) , methyl ester, Octadec-9enoic acid, cis-11,14-Eicosadienoic acid, methyl ester and (Z)-18-Octadec-9enolide. Main reactions involved in this conversion include isomerization, dehydrogenation and reduction as confirmed by the in silico analysis. The putative linoleate isomerase and dehydrogenase catalyzing the relevant reactions were identified using the whole genome sequence of $L$. plantarum 13-3. These two enzymes were shown to bind the LA molecule at their active sites mainly by formation of hydrogen bonding between the acidic group of LA and the proline residues of the enzymes. Further study is required to investigate the role of the conversion from LA to its various fatty acid metabolites by $L$. plantarum 13-3 physiologically and the implications for exploring the potentiality in functional foods.

\section{ACKNOWLEDGEMENTS}

Financial support from Natural Science Foundation of China (No. 31871823) and Beijing Talent Cultivation Quality Construction - First-class Professional Construction (Municipal Level) - Food Science and Engineering (PXM2019_014213_000010) was kindly acknowledged.

\section{REFERENCES:}

1. Wang R, Kern JT, Goodfriend TL, Ball DL, Luesch H. Activation of the antioxidant response element by specific oxidized metabolites of linoleic acid. Prostaglandins Leukot. Essent. Fatty Acids. 81, 5359, (2009).

2. Cunnane SC, Guesnet P. Linoleic acid recommendations-a house of cards. Prostaglandins Leukot. Essent. Fatty Acids. 85, 399402, (2011).

3. Mathieu P, Pibarot P, Despres JP. Metabolic syndrome: the danger signal in atherosclerosis. Vasc. Health Risk Manag .2, 285302, (2006).

4. Niki E. Lipid peroxidation: physiological levels and dual biological effects. Free Radic. Biol. Med. 47, 469484, (2009).

5. Tavakoli Yaraki, M Karami Tehrani F. Apoptosis Induced by 13hydroxyoctadecadienoic acid in the breast cancer cell lines, MCF-7 and MDA-MB-231. Iran. J. Basic Med. Sci. 16, 653659, (2013).

6. Vangaveti V, Baune BT, Kennedy RL. Hydroxyoctadecadienoic acids: novel regulators of macrophage differentiation and atherogenesis. Ther. Adv. Endocrinol. Metab. 1, 5160, (2010).

7. Yuan H, Li MY, Ma LT, et al. 15-Lipoxygenases and its metabolites 15(S)HETE and 13(S)-HODE in the development of non-small cell lung cancer Thorax 65, 321326, (2010).

8. J.F. Greene, B.D. Hammock, Toxicity of linoleic acid metabolites, in: K.V. Honn, L.J. Marnett, S. Nigam, C.N. Serham, E.A. Dennis (Eds.), Eicosanoids and Other Bioactive Lipids in Cancer, Inflammation, and Radiation Injury, Kluwer Academic/Plenum Press, New York. 471477, (1999).

9. M.R. Buchanan, Linoleic acid metabolites in health and disease, Adv. Exp. Med. Biol. 469, 463469, (1999).

10. Sonnenburg, JL.; Backhed, F. Diet-microbiota interactions as moderators of human metabolism. Nature, 535, 7610,664, (2016).

11. Marc Schoeler.; Robert Caesar. Dietary lipids, gut microbiota and lipid metabolism Reviews in Endocrine and Metabolic Disorders 112, (2019).

12. DL, Greenway.; KG, Dyke. Mechanism of the inhibitory action of linoleic acid on the growth of Staphylococcus aureus. J. Gen. Microbiol. 115, 233245, (1979).

13. MK, Raychowdhury.; R, Goswami.; P, Chakrabarti. Effect of unsaturated fatty acids in growth inhibition of some penicillin-resistant and sensitive bacteria, J. Appl. Bacteriol. 59, 183188, (1985).

14. H. Keweloh.; H.J. Heipieper. Trans unsaturated fatty acids in bacteria, Lipids 31, 129137, (1966).

15. CJ, Zheng.; JS Yoo.; TG Lee.; HY Cho.; YH Kim.; WG Kim. Fatty acid synthesis is a target for antibacterial activity of unsaturated fatty acids. FEBS Lett. 579, 51575162, (2005).

16. A, Buccioni.; M, Decandia.; S, Minieri.; G, Molle.; A, Cabiddu. Lipid metabolism in the rumen: new insights on lipolysis and biohydrogenation with an emphasis on the role of endogenous plant factors, Anim. Feed Sci. Technol. 174,125, (2012).

17. MR, Maia LC.; Chaudhary.; L, Figueres.; RJ, Wallace. Metabolism of polyunsaturated fatty acids and their toxicity to the microflora of the rumen. Antonie Van Leeuwenhoek 91, 303314, (2007).

18. N, McKain.; KJ, Shingfield.; RJ, Wallace. Metabolism of conjugated linoleic acids and 18: 1 fatty acids by ruminal bacteria: products and mechanisms, Microbiol-Sgm. 156579-588, (2010). 
19. IS, Nam.; PC, Garnsworthy. Biohydrogenation of linoleic acid by rumen fungi compared with rumen bacteria. J. Appl. Microbiol. 103, 551556, (2007).

20. Selhub EM, Logan AC, Bested AC, Fermented foods, microbiota, and mental health: ancient practice meets nutritional psychiatry. J. Physiol. Anthropol. 33, 112, (2014).

21. Shah NP, Functional cultures and health benefits. Int. Dairy J.17, 12621277 , (2007).

22. Younesi E, Ayseli MT. An integrated systems-based model for substantiation of health claims in functional food development. Trends Food Sci. Tech. 41,95100, (2015)

23. S. Torriani, F. Clementi, M. Vancanneyt, B. Hoste, F. Dellaglio, and K. Kersters, "Differentiation of Lactobacillus plantarum, L. pentosus and L. paraplantarum species by RAPD-PCR and AFLP," Systematic and Applied Microbiology, 24, 4, 554560, (2001).

24. E. A. Pfeiler and T. R. Klaenhammer, "The genomics of lactic acid bacteria," Trends in Microbiology, 15, 12, 546553, (2007).

25. R. J. Siezen, V. A. Tzeneva, A. Castioni et al., "Phenotypic and genomic diversity of Lactobacillus plantarum strains isolated from various environmental niches," Environmental Microbiology, 12,3, 758773, (2010).

26. R. J. Siezen and J. E. T. van Hylckama Vlieg, "Genomic diversity and versatility of Lactobacillus plantarum, a natural metabolic engineer," Microbial Cell Factories. 10, S3, 1, (2011).

27. J. Wang, H. Ji, D. Zhang et al., "Assessment of probiotic properties of Lactobacillus plantarum ZLP001 isolated from gastrointestinal tract of weaning pigs," African Journal of Biotechnology. 10,54, 1130311308, (2011).

28. Y. Nami, N. Abdullah, B. Haghshenas, D. Radiah, R. Rosli, and A. Y. Khosroushahi, "Assessment of probiotic potential and anticancer activity of newly isolated vaginal bacterium Lactobacillus plantarum 5BL," Microbiology and Immunology, 58, 9, 492502, (2014).

29. R. Tabasco, F. Sánchez-Patán, M. Monagas et al., "Effect of grape polyphenols on lactic acid bacteria and Bifidobacteria growth: resistance and metabolism," Food Microbiology, 28,7,13451352, (2011).

30. P. Fras, F. M. Campos, T. Hogg, and J. A. Couto, "Production of volatile phenols by Lactobacillus plantarum in wine conditions. Biotechnology Letters. 36, 2, 281285, (2014).

31. E. J. Yang and H. C. Chang, "Purification of a new antifungal compound produced by Lactobacillus plantarum AF1 isolated from kimchi," International Journal of Food Microbiology. 139,12,5663, (2010).

32. P. Li, Q. Zhou, and Q. Gu, "Complete genome sequence of Lactobacillus plantarum LZ227, a potential probiotic strain producing B-group vitamins," Journal of Biotechnology. 234, 6670, (2016).

33. K. B. Ahn, J. B. Baik, O. Park, C. Yun, and S. H. Han, "Lactobacillus plantarum lipoteichoic acid inhibits biofilm formation of Streptococcus mutans," PLoS One. 13, 2, e0192694, (2018).

34. S, Kishino.; J, Ogawa.; K, Yokozeki.; S, Shimizu. Linoleic acid isomerase in Lactobacillus plantarum AKU1009a proved to be a multi-component enzyme system requiring oxidoreduction cofactors. Biosci. Biotechnol. Biochem. 75,318322, (2011a)
35. S. Kishino.; SB, Park.; M, Takeuchi.; K, Yokozeki.; S, Shimizu.; J, Ogawa Novel multicomponent enzyme machinery in lactic acid bacteria catalyzing $\mathrm{C}=\mathrm{C}$ double bond migration useful for conjugated fatty acid synthesis. Biochem. Biophys. Res. Commun. 416: 188193, (2011b).

36. S, Kishino.; M, Takeuchi.; SB, Park.; A, Hirata.; N, Kitamura.; J, Kunisawa.; H, Kiyono.; R, Iwamoto.; Y, Isobe.; M, Arita.; H, Arai.; K, Ueda.; J, Shima.; S, Takahashi.; K, Yokozeki.; S, Shimizu.; J, Ogawa. Polyunsaturated fatty acid saturation by gut lactic acid bacteria affecting host lipid composition Proc. Natl. Acad. Sci. USA. 110, 1780817813, (2013)

37. Barrett, E.; Ross, RP.; Fitzgerald, GF.; Stanton, C. Rapid screening method for analyzing the conjugated linoleic acid production capabilities of bacterial cultures. Appl. Environ. Microbiol. 73, 7,23332337,(2007).

38. Shantha, N.; Decker, EA.; Henning, B. Comparison of methylation methods for the quantification of Conjugated Linoleic Isomer. J. AOAC Int. 76,664649, (1993).

39. Biasini, M.; Bienert, S.; Waterhouse, A.; Arnold, K.; Studer, G.; Schmidt, T SWISS-MODEL: modelling protein tertiary and quaternary structure using evolutionary information., Nucleic Acids Research. 42, W252, 8, (2014).

40. Dassault Systèmes BIOVIA, Discovery Studio Modeling Environment. (2015).

41. Hanwell, MD.; Curtis, DE.; Lonie, DC.; Vandermeerschd, T.; Zurek, E.; Hutchison, GR.; Avogadro. An advanced semantic chemical editor, visualization, and analysis platform. J. Cheminform. 4, 117, (2012).

42. Morris, GM.; Huey, R.; Lindstrom, W.; Sanner, MF.; Belew, RK.; Goodsell, DS. AutoDock4 and AutoDockTools4: Automated docking with selective receptor flexibility. J. Comput. Chem. 30, 278591, (2009).

43. Kim YJ, Liu RH, Bond DR, Russell JB. Effect of linoleic acid concentration on conjugated linoleic acid production by Butyrivibrio fibrisolvens A38. Appl. Environ. Microbiol. 66, 12, 52265230, (2000).

44. van Nieuwenhove CP, Oliszewski R, Gonzalez SN, Perez Chaia AB.Conjugated linoleic acid conversion by dairy bacteria cultured in MRS broth and buffalo milk. Lett. Appl. Microbiol. 44, 5,467474, (2007)

45. Ogawa J, Kishino S, Ando A, Sugimoto S, Mihara K, Shimizu S. Production of conjugated fatty acids by lactic acid bacteria. J. Biosci. Bioeng. 100, 4, 355364, (2005).

46. Chung SH, Kim IH, Park HG, Kang HS, Yoon CS, Jeong HY, Choi NJ, Kwon EG, Kim YJ. Synthesis of conjugated linoleic acid by human-derived Bifido bacterium breve LMC 017: utilization as a functional starter culture for milk fermentation. J. Agric. Food Chem. 56,9, 33113316, (2008).

47. Tung Y Lin, Chin-Wen Lin, Chien-Hsing Lee. Conjugated linoleic acid concentration as affected by lactic cultures and added linoleic acid Food Chemistry. 67, 1, 5, (1999).

48. Alonso L, Cuesta EP, Gilliand SE. Production of free conjugated linoleic acid by Lactobacillus acidophilus and Lactobacillus casei of human intestina origin. J. Dairy Sci. 86, 6, 19411946, (2003). 